\title{
Stickler Syndrome
}

National Cancer Institute

\section{Source}

National Cancer Institute. Stickler Syndrome. NCI Thesaurus. Code C74984.

A rare autosomal dominant syndrome caused by mutations in the COL11A1, COL11A2, and COL2A1 genes which affect the production of type II and XI collagen. It is characterized by a range of signs and symptoms including cleft palate, large tongue, small lower jaw, hearing loss, myopia, glaucoma, retinal detachment, skeletal, and joint abnormalities. 